\title{
Oxytocin Does Not Attenuate the ex vivo Production of Inflammatory Cytokines by Lipopolysaccharide-Activated Monocytes and Macrophages from Healthy Male and Female Donors
}

\author{
Kharah M. Ross Gaye McDonald-Jones Gregory E. Miller
}

Northwestern University, Evanston, III., USA

\section{Key Words}

Oxytocin - Monocytes · Macrophages - Lipopolysaccharide · Inflammation · Humans

\begin{abstract}
Background: Oxytocin (OT) is a neuropeptide shown to attenuate inflammatory responses in both humans and animals, but the specific mechanism underlying these actions has not yet been identified. Preliminary research in humans suggests that monocytes (MOs) and macrophages (MPs) could be the target of anti-inflammatory actions of OT. Here, we present a series of ex vivo experiments in human MOs and MPs, testing whether OT attenuates the cytokine responses of these cells to a common bacterial product, lipopolysaccharide (LPS). Methods: MO experiments were conducted using blood samples taken from healthy volunteers after obtaining informed consent. MPs were purchased frozen from a cell supplier. All samples were cultured under standard conditions: for $6 \mathrm{~h}$ at $37^{\circ} \mathrm{C}$ in a $5 \% \mathrm{CO}_{2}$ atmosphere. A number of variables were considered: volunteer sex, method of MO isolation, LPS concentration, OT concentration, preincubation with OT, cytokines measured, and method of cytokine measurement. Results: Regardless of the specific conditions, no attenuation of LPS-stimulated cytokine production by OT was observed in either MOs or MPs. Conclu-
\end{abstract}

sion: OT does not attenuate MO or MP inflammatory cytokine production following LPS stimulation. The previously observed anti-inflammatory properties of OT may be attributable to effects on other classes of immune cells or actions in other lymphoid compartments. Alternatively, the effects of OT on inflammation could be secondary to other neurohormonal changes it elicits.

Copyright $\odot 2013$ S. Karger AG, Basel

\section{Introduction}

Oxytocin (OT) is a nonapeptide neurotransmitter, hormone produced and secreted centrally, by the hypothalamus and the posterior pituitary, and also peripherally, by reproductive organs, the thymus and the adrenal glands [1]. At least three general physiological roles have emerged for OT: it is best characterized for its classical role in regulating parturition and lactation [2] and increasingly recognized as having a role in social interactions and social bonding [2-5]. Recent research has highlighted a third novel role of OT in regulating inflammatory responses. This has been observed across a wide range of inflammatory disease models in animals, including sepsis and infection $[6,7]$, atherosclerosis [8, 9], musculocutaneous flap survival [10], organ damage and in-

\section{KARGER}

E-Mail karger@karger.com

www.karger.com/nim
(C) 2013 S. Karger AG, Basel

1021-7401/13/0205-0285\$38.00/0
Kharah M. Ross, MA

Department of Psychology 2029 Sheridan Rd

Evanston, IL 60208-2710 (USA)

E-Mail kmross@u.northwestern.edu 
flammation [11-14], and burns [15, 16]. In human models, OT has been shown to reduce systemic inflammation in a model of sepsis [17]. Given its systemic anti-inflammatory effects, OT is increasingly being considered as a potential therapeutic agent for inflammatory diseases but, as of yet, there is no agreement as to how and where $\mathrm{OT}$ is acting.

In humans, the monocyte (MO)/macrophage (MP) cell line has been singled out as a possible mechanistic link, which is especially of therapeutic interest given that MPs and their precursors, MOs, play a key role in initiating and sustaining inflammatory processes that contribute to chronic diseases, including depression, atherosclerosis, neurodegenerative disorders, and some cancers [18, 19]. To date, two studies have explored the anti-inflammatory potential of OT in humans and assessed whether any effect could be mediated by the action of OT on MOs and/or MPs. The first study by Szeto et al. [20] confirmed that an OT receptor (OTR) could be detected on healthy peripheral blood mononucleocytes and THP-1 MOs, isolated from a 1-year-old Japanese boy with acute monocytic leukemia [21], and derived MPs, suggesting that OT is in fact capable of directly regulating these cells. THP1-derived MPs were then activated with lipopolysaccharide (LPS), a molecule found on the surface of Gram-negative bacteria, and cocultured with $0-100$ pM OT for $6 \mathrm{~h}$ under standard conditions. A clear attenuation of interleukin-6 (IL-6) secretion by the THP-1 MPs was observed, suggesting that MOs and MPs may be involved in the systemic anti-inflammatory effects of OT. This contrasts with the results of the second study by Clodi et al. [17], which consisted of a randomized, placebo-controlled cross-over design where 10 healthy young males were given an intravenous bolus of LPS concurrent with administration of OT. Again, OT was shown to attenuate the systemic inflammatory cytokine response. However, when 4 samples of peripheral MOs were preincubated ex vivo with $0-100 \mathrm{nM}$ OT for $30 \mathrm{~min}$, then activated with LPS and cultured for 2, 4 or $24 \mathrm{~h}$, no attenuation of tumor necrosis factor- $\alpha$ (TNF- $\alpha$ ), MO chemoattractant protein-1 or IL-6 production was observed. As such, these results suggest that although OT has systemic anti-inflammatory effects in male humans, MOs do not appear to mediate that effect.

These studies provide conflicting evidence about whether peripheral MOs and MPs are involved in the systemic anti-inflammatory effects of OT in humans. Furthermore, the work to date has been done exclusively in male cells, so it is not known if these results extend to cells isolated from females. Given that there are gender differ- ences with respect to OT activity and function $[2,22]$, it is important to determine whether any effects of OT are observable in cells from both male and female donors. Existing research has also focused on cell-line MPs, which may behave differently from primary MPs from healthy donors. Resolving these issues would have implications for our more general understanding of neuroimmune interactions and for the potential use of OT as an anti-inflammatory therapeutic agent. As such, the first goal of our experiments was to assess the viability of this particular mechanism, i.e. to determine whether or not OT clearly regulates inflammatory cytokine production in MOs and MPs taken from a sample of healthy human participants. The second goal of our experiments was to extend the work of Clodi et al. [17] and Szeto et al. [20] by assessing the potential anti-inflammatory effect of OT in MOs isolated from females and in primary MPs from healthy humans. Finally, previous research measured the quantified amount of cytokines secreted into the supernatant, but it is possible that intracellular cytokine production can be altered without a corresponding change in cytokine secretion. As such, we also incorporated intracellular staining and flow cytometry into our methods in order to differentiate between cytokine production and cytokine secretion. If OT does clearly affect MO and MP inflammatory cytokine secretion, we would expect to see a clear dose-response curve showing attenuation of cytokine production and/or secretion by increasing concentration of OT.

\section{Methods}

Cells

For experiments with MOs, whole blood was collected from healthy male and female volunteers, from 20 to 30 years of age, who provided informed consent and were compensated CAD 10 for participating. Most OT inflammation research focuses on male animals or human participants in order to avoid complications arising from hormone changes that occur during the menstrual cycle. However, if OT is to be used therapeutically, as an anti-inflammatory agent, we must know if its effects are gender dependent. As such, both sexes were included. Blood was taken via antecubital blood draw by a trained phlebotomist into, unless otherwise stated, 5-ml sodium heparin Vacutainer tubes (BD Biosciences, Mississuaga, Ont., Canada). All whole-blood samples were diluted 10:1 with saline prior to culturing. For MP experiments, we purchased MO-derived, in vitro matured MPs from a cell supplier (AllCells, Emeryville, Calif., USA). All MPs used here were matured from a single donor's peripheral MOs. Following the supplier's instructions, cells were thawed, checked for viability and stabilized for $24-72 \mathrm{~h}$ prior to use in the cultures. All procedures and methods were approved by the University of British Columbia Research Ethics Board. 
Table 1. Summary of experimental protocols tested

\begin{tabular}{|c|c|c|c|c|c|c|}
\hline 1 (fig. 1) & female & monocytes (whole blood) & 50 & $0,1,10,100,1,000$ & 0 & FACS (CD14+/IL-6+) \\
\hline \multirow[t]{2}{*}{2 (fig. 1) } & female & monocytes (whole blood) & 50 & $0,1,10,100,1,000$ & 0 & FACS (CD14+/IL-6+) \\
\hline & & monocytes (whole blood) & 50 & $0,1,10,100,1,000$ & 30 & FACS (CD14+/IL-6+) \\
\hline \multirow[t]{2}{*}{3 (fig. 1) } & male & isolated PBMCs & 50 & $0,1,10,100,1,000$ & 30 & FACS (CD14+/IL-6+) \\
\hline & female & isolated PBMCs & 50 & $0,1,10,100,1,000$ & 30 & FACS (CD14+/IL-6+) \\
\hline \multirow[t]{2}{*}{4 (fig. 2) } & female & monocytes (whole blood) & 50 & $0,1,10,100,1,000$ & 0 & ELISA (IL-6) \\
\hline & & monocytes (whole blood) & 0.5 & $0,1,10,100,1,000$ & 0 & ELISA (IL-6) \\
\hline \multirow[t]{4}{*}{7 (fig. 2, 4) } & male & macrophages & 50 & $0,0.1,10,100,1,000$ & 0 & $\begin{array}{l}\text { ELISA (TNF- } \alpha \text { ); } \\
\text { Meso Scale (TNF- } \alpha \text {, IL-6, IL-8, IL-1 } \beta \text { ) }\end{array}$ \\
\hline & male & macrophages & 50 & $0,0.1,10,100,1,000$ & 30 & $\begin{array}{l}\text { ELISA (TNF- } \alpha \text { ); } \\
\text { Meso Scale (TNF- } \alpha \text {, IL-6, IL-8, IL-1 } \beta \text { ) }\end{array}$ \\
\hline & male & macrophages & 50 & $0,0.1,10,100,1,000$ & 0 & Meso Scale (TNF- $\alpha$, IL-6, IL-8, IL-1 $\beta$ ) \\
\hline & male & macrophages & 50 & $0,0.1,10,100,1,000$ & 30 & Meso Scale (TNF- $\alpha$, IL-6, IL-8, IL-1 $\beta$ ) \\
\hline
\end{tabular}

PBMCs = Peripheral blood mononuclear cells.

\section{Culturing}

Unless otherwise indicated, all culturing was done using flatbottomed tissue culture plates with incubations lasting $6 \mathrm{~h}$ at $37^{\circ} \mathrm{C}$ in $5 \% \mathrm{CO}_{2}$, similar conditions for which positive results were reported by Szeto et al. [20]. For experiments with MOs, 1,600 $\mu$ l of saline-diluted whole blood was added to each well, corresponding to roughly $6.2 \times 10^{5} \mathrm{MOs}$. For MP experiments, a cell counter was used to titrate suspensions to $5.0 \times 10^{5}$ cells in $800 \mu \mathrm{l}$ of R10 medium per well. A 'standard range' of OT concentrations, 10-1,000 pM, was selected to encompass normal physiological levels $[23,24]$. However, some experiments included what would be considered supraphysiological levels (1,000-10,000 pM OT). As such, OT concentrations in our experiments encompassed the whole range used in both the analyses of Szeto et al. [20] and Clodi et al. [17]. Unless otherwise stated, cell cultures were stimulated with $50 \mathrm{ng} / \mathrm{ml} \mathrm{LPS}$.

\section{Cytokine Measurement}

Three methods were used to quantify cytokine synthesis and release: intracellular cytokine staining (ICS) using fluorescenceactivated cell sorting (FACS; FACSCalibur, BD Biosciences), enzyme-linked immunosorbent assay (ELISA; R\&D Systems, Minneapolis, Minn., USA) and electrochemiluminescence (ECL) on a Meso Scale Discovery multiplexing instrument (Sector Imager 2400, Gaithersburg, Md., USA).

ICS was used to quantify intracellular cytokine production in response to LPS stimulation [25]. In these experiments, whole blood was cocultured with LPS and OT at varying doses under varying conditions (table 1 ) for $6 \mathrm{~h}$. Each well also included a $0.1 \%$ Brefeldin A solution (BD Biosciences), a Golgi plug that prevents cytokine secretion from the cell. Following incubation, cultures were stored at $4^{\circ} \mathrm{C}$ over night and stained for ICS analysis the next day. All staining incubations were done at room temperature in the dark. First, red blood cells were lysed (Pharmlyse, BD Biosciences) and removed by washing, followed by resuspension in staining buffer ( $1 \%$ fetal bovine serum in phosphate-buffered saline; BD Biosciences). Fc receptors were blocked by incubating for 15 min with $10 \%$ normal human serum. Cells were stained with CD-14 (10\% APC-conjugated CD-14 Ab; BD Biosciences) during a 20-min incubation, followed by a wash with staining buffer. Cells were fixed and permeabilized using a Cytofix/Cytoperm Plus Fixation/Permeabilization kit (BD Biosciences), as per manufacturer's instructions. Cells were then stained for intracellular IL-6 (4\% phycoerythrin-conjugated IL-6 antibody; BD Biosciences) during a 20-min incubation. The samples were washed again and resuspended in staining buffer prior to analysis on a FACSCalibur (BD Biosciences). Data were analyzed with FlowJo (Tree Star Inc., Ashland, Oreg., USA). Quadrants were set using data from concurrently run negative control samples (unstimulated and nonstained samples), and positive and negative beads stained with appropriate antibodies.

ELISA and ECL were used to assess cytokine release into the supernatant. For both assays, whole blood was cocultured with LPS 
and $\mathrm{OT}$ at varying doses under varying conditions (table 1 ) for $6 \mathrm{~h}$. Following incubation, each well was centrifuged and the supernatant was harvested and stored at $-30^{\circ} \mathrm{C}$. MO cytokine production was analyzed using a human IL-6 DuoSet ELISA kit (R\&D Systems). Plates were coated with antibody during the afternoon, left overnight at room temperature and blocked at least $1 \mathrm{~h}$ prior to assay. Plates were washed three times prior to adding sample and standards. Standards were prepared and diluted as per kit instructions (600-9.38 pg/ml). Supernatant samples were thawed immediately before assay, vortexed and centrifuged to remove any remaining solids and underwent a $400 \times$ dilution. Both standards and samples were plated in duplicate. Plates were read at $450 \mathrm{~nm}$ on a Tecan Sunrise plate reader (Medford, Mass., USA). The average intra-assay coefficient of variation $(\mathrm{CV})$ for these analyses was $6.12 \%$.

In some experiments, we also quantified MP production of TNF- $\alpha$ using the Quantikine ELISA kit (BD Biosciences). As above, samples were thawed, vortexed and centrifuged immediately prior to assay. The manufacturer's instructions were followed at each step and the plates were subsequently read at $490 \mathrm{~nm}$ on the Tecan Sunrise plate reader. Average CVs for these analyses were $7.36 \%$.

MP supernatant samples and some MO samples were also assessed using ECL in order to obtain multiplex readouts for multiple cytokines. A Human Proinflammatory-4 II Tissue Culture kit containing prepared plates stained for IL-6, IL-8, IL- $1 \beta$ and TNF- $\alpha$ was purchased from Meso Scale Discovery. Samples were analyzed neat following the manufacturer's instructions on a Sector instrument. Average intra-assay CVs for MO analyses were $11.2 \%$ for IL-8, 5.66\% for TNF- $\alpha, 3.69 \%$ for IL-6 and $6.09 \%$ for IL- $1 \beta$. Average intra-assay CVs for MP analyses were $4.64 \%$ for IL- $8,1.54 \%$ for TNF- $\alpha, 5.12 \%$ for IL- 6 and $14.2 \%$ for IL- $1 \beta$.

\section{Ligands}

OT obtained from Cedarlane (Burlington, Ont., Canada), the same supplier used for the experiments in Szeto et al. [20], was diluted and prepared according to the manufacturer's instructions. $\mathrm{OT}$ aliquots were stored at $-80^{\circ} \mathrm{C}$, thawed and diluted to working concentrations prior to cell culturing. LPS was obtained from Sigma-Aldrich (Oakville, Ont., Canada) and stored at $-30^{\circ} \mathrm{C}$. Unless otherwise specified, LPS was diluted to a final concentration of $50 \mathrm{ng} / \mathrm{ml}$ prior to culturing.

\section{Results}

\section{Experiment 1}

The goal of the first experiment was to determine if OT could be observed to modulate proinflammatory cytokine production by MOs stimulated with LPS. Wholeblood samples were taken from a male and female volunteer, diluted 10:1 with saline solution, cultured with LPS and standard OT concentrations and incubated as described above (please refer to table 1 for a summary of all experimental protocols). After incubation, production of IL-6 was measured by ICS. Results were graphed and visually inspected for an attenuation curve. As can be seen

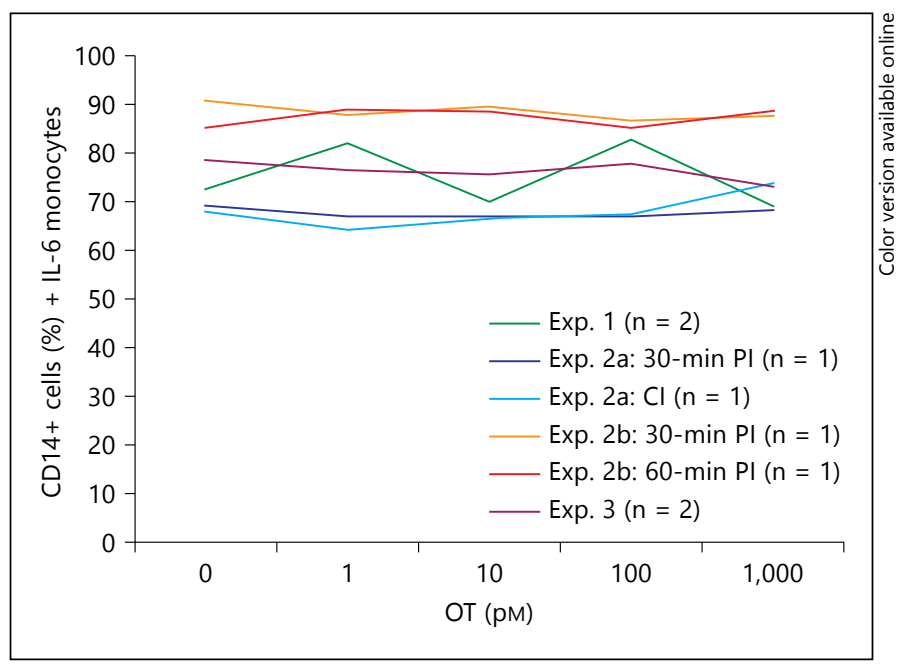

Fig. 1. MO ICS data showing the results from experiments 1,2 and 3. Experiment 1: male and female whole-blood MOs incubated concurrently with $50 \mathrm{ng} / \mathrm{ml}$ LPS and 0-1,000 pM OT, analyzed via FACS. Experiment 2: male and female whole-blood MOs incubated with $50 \mathrm{ng} / \mathrm{ml} \mathrm{LPS}$, either concurrently or following a 30- or 60-min preincubation with $0-1,000$ pM OT, analyzed via FACS. Experiment 3: male and female isolated peripheral blood mononuclear cells incubated with $50 \mathrm{ng} / \mathrm{ml}$ LPS following a 30-min preincubation with 0-1,000 nM OT, analyzed via FACS. PI = Preincubation; $\mathrm{CI}=$ concurrent incubation; $\mathrm{n}=$ number of participants per condition. Data are expressed as percentage of CD14+ cells that also stain IL-6+.

in figure 1, the curve was flat, suggesting that under these conditions, OT did not attenuate LPS-stimulated IL-6 production in whole-blood MOs, regardless of participant sex.

\section{Experiment 2}

It was possible that no attenuation was observable in experiment 1 because MOs may need to be exposed to OT before stimulation occurs. This would be the case if OT regulates how cells prepare for challenge instead of the challenge response itself. To test this, we repeated experiment 1 but introduced periods of preincubation with OT prior to stimulating MOs with LPS.

Two whole-blood samples were taken from a female volunteer. Both samples were cultured as in experiment 1 , except the second sample was preincubated with OT for 30 min before adding LPS (experiment 2a). IL-6 production was again analyzed via ICS, and the results were graphed and inspected. Again, no attenuation was observed. Another two whole-blood samples were taken from a different female volunteer and cultured as in experiment 1 , except one sample was preincubated with OT 
for $30 \mathrm{~min}$ and the other for $60 \mathrm{~min}$ (experiment 2b). Inspection of FACS data again showed no clear attenuation of IL-6 production by OT in MOs (fig. 1).

\section{Experiment 3}

Prior research on OT regulation of uterine contractions suggests that calcium may play a role in OT signal transduction [26]. All our previous experiments used whole blood collected in a sodium heparin tube, which prevents clotting by chelating calcium ions. As such, it was possible that no action of OT had been observed because a key signal transducer was not available in culture. To assess this possibility, MOs were isolated from whole blood and cultured in a cell medium that replaced depleted calcium.

Blood samples were taken from a male and female volunteer using 8-ml CPT Vacutainer tubes (BD Biosciences). Within $2 \mathrm{~h}$ of the blood draw, the tubes were centrifuged for $20 \mathrm{~min}$ at $1,800 \mathrm{RCF}$. Mononuclear cells present at the interface were harvested, washed in RPMI-1640 and then resuspended in R10, a cell medium that contains calcium and other nutrients, at a final concentration of $10^{6}$ cells $/ \mathrm{ml}$. Samples were pretreated with varying OT doses (table 1) for 30 min prior to stimulation with LPS and then incubated as usual for $6 \mathrm{~h}$. Following incubation, the samples were prepared for ICS, analyzed by FACS, and results were graphed and visually inspected for attenuation of IL-6 production (figure 1). Again, no attenuation of IL- 6 production by OT concentration was observed, regardless of participant sex, suggesting that null results observed using whole-blood samples was not because a key signal transduction molecule had been removed during sampling.

\section{Experiment 4}

ICS indexes cytokine production but not cytokine release. Since it is plausible that the anti-inflammatory properties of OT involve modulation of the latter process, we repeated experiment 1 but assessed the magnitude of cytokine secretion into the culture supernatant by ELISA (experiment 3a). We also tested whether the relatively high dose of LPS used in earlier experiments might have 'washed out' any effects of OT. As such, here we also repeated experiment 1 but used a 100 -fold lower dose of LPS ( $0.5 \mathrm{ng} / \mathrm{ml}$; experiment $3 \mathrm{~b})$.

Two whole-blood samples were taken from a female volunteer. The first sample was cultured as in experiment 1, except no Golgi plug was added to the culture, allowing the MOs to freely secrete cytokines into the culture medium. The second sample was also cultured the same way

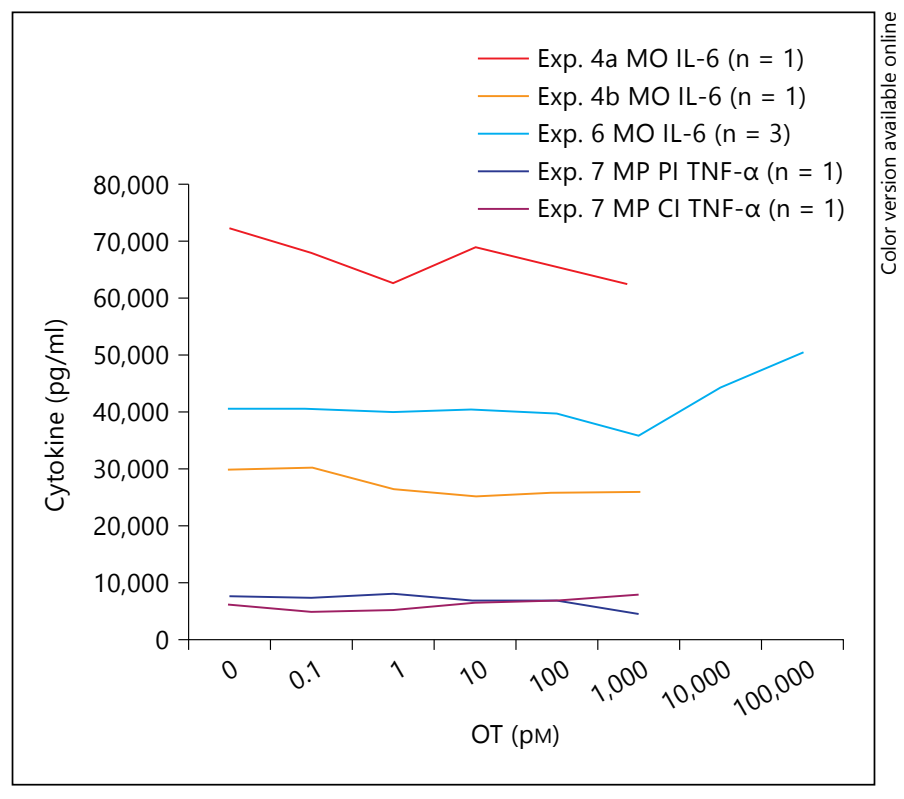

Fig. 2. MO and MP ELISA data showing results from experiments 4, 6 and 7. Experiment 4: female whole-blood MOs incubated concurrently with $0-1,000$ pM OT and either 50 or $0.5 \mathrm{ng} / \mathrm{ml}$ LPS secreted IL-6 analyzed via ELISA. Experiment 6: male and two female whole-blood MOs incubated with $50 \mathrm{ng} / \mathrm{ml}$ LPS following a 30-min preincubation with 0-10,000 nM OT secreted IL-6 analyzed via ELISA. Experiment 7: male MPs incubated with $50 \mathrm{ng} / \mathrm{ml}$ LPS either concurrently or following a 30-min preincubation with $0-1,000$ pM OT, secreted TNF- $\alpha$ analyzed via ELISA. PI $=30-\mathrm{min}$ preincubation; $\mathrm{CI}=$ concurrent incubation; $\mathrm{n}=$ number of participants per condition.

except diluted LPS was used to challenge the MOs. Culture supernatant IL- 6 was measured using ELISA, and the results were graphed and visually inspected for an attenuation curve. Again, no attenuation of IL-6 secretion by OT in MOs was observed. When the concentration of LPS was reduced, overall IL- 6 secretion decreased but no attenuation curve emerged (fig. 2).

\section{Experiment 5}

After the results of experiment 3, we concluded that LPS concentration was likely not masking any effect of OT and so we returned to the original LPS concentration. Again, it was possible that MOs needed to be preincubated with OT if this hormone regulates cell readiness for challenge. It is also possible that OT might not regulate MO IL- 6 but other proinflammatory mediators instead.

To test these possibilities, a whole-blood sample was taken from a male volunteer and preincubated with OT for $30 \mathrm{~min}$ prior to LPS challenge. Culture supernatant was analyzed by mutiplex ECL and results were graphed. 
As can be seen in figure 3, under these conditions, OT did not attenuate MO secretion of IL-6, nor did it modulate LPS-stimulated production of other key proinflammatory mediators including IL-8, TNF- $\alpha$ and IL- $1 \beta$.

\section{Experiment 6}

In the previous experiments, we used OT doses within the physiological range for healthy adults. However, under certain circumstances, such as during birth, the circulating concentration of OT is much higher. It is possible that MOs only respond to OT under these supraphysiologic conditions. To test this notion, we stimulated MOs with a broader range of OT concentrations.

Whole-blood samples were taken from a male and female volunteer. Samples were cultured with LPS under typical conditions but the OT concentrations used were $0,0.1,1,10,100,1,000$ and 10,000 pM (table 1). Culture supernatant IL- 6 was measured using ELISA and graphed for visual inspection (fig. 2). Again, no clear pattern of IL-6 secretion attenuation by OT was observed, regardless of participant sex, even at the higher concentrations of OT.

\section{Experiment 7}

Based on the results of experiments 1-6, which included cells from 8 different individuals, we concluded that OT does not act directly on primary human MOs to attenuate LPS-induced cytokine synthesis or release. However, as prior work shows that OTR concentration is much denser on the surface of MPs than of MOs [20], we decided to reconduct some of these studies with healthy human MPs on the assumption that these mature cells might be more OT responsive.

Thawed MPs were cultured with LPS, with OT coincubation or preincubation at varying doses (table 1). Culture supernatant TNF- $\alpha$ was assessed via ELISA, and results were graphed and inspected for an attenuation curve. Again, no clear curve emerged (fig. 2).

These MP experiments were repeated in another set of studies, but multiplex ECL methods were used to determine if other cytokines might be affected. As figure 4 shows, OT had no discernable influence on LPS-stimulated MP production of IL-6, TNF- $\alpha$, IL-8 and IL- $1 \beta$.

\section{Discussion}

The purpose of this study was to assess whether OT can inhibit MO and/or MP cytokine production following activation with LPS and to extend previous research

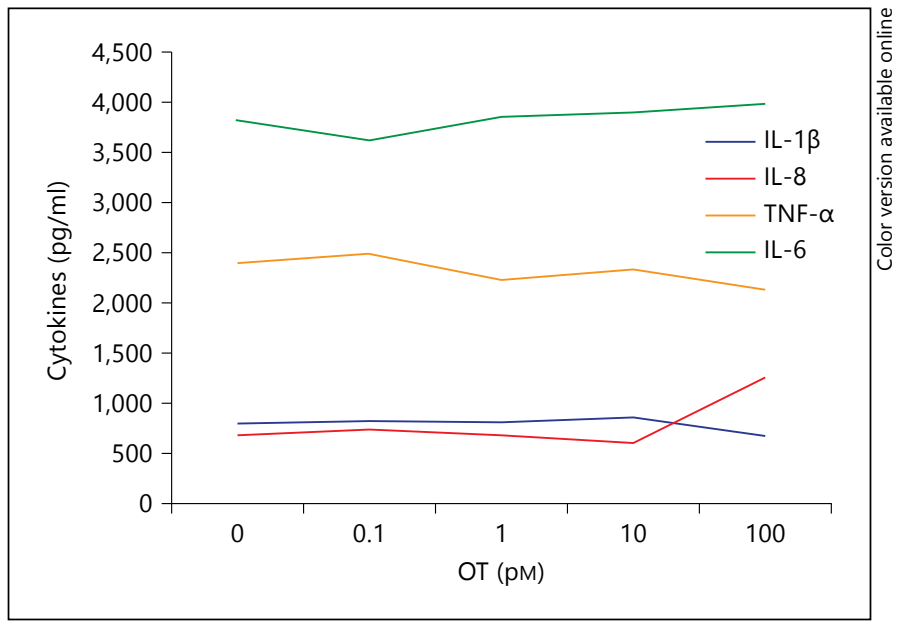

Fig. 3. MO ECL data showing the results from experiment 5: male whole-blood MOs incubated with $50 \mathrm{ng} / \mathrm{ml}$ LPS following a 30min preincubation with $0-100$ pM OT secreted cytokines (TNF- $\alpha$, IL-6, IL-8 and IL-1 $\beta)$ analyzed via Meso Scale $(n=1)$.

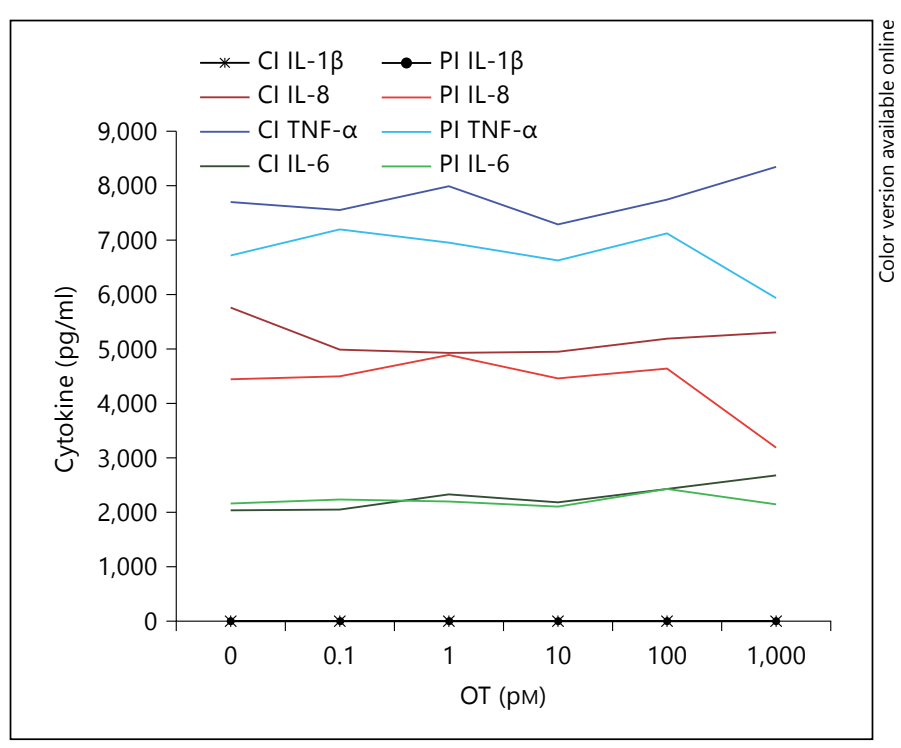

Fig. 4. MP ECL data, showing the results from experiment 7: two separate MP samples from the same male incubated with $50 \mathrm{ng} / \mathrm{ml}$ LPS either concurrently or following a 30-min preincubation with $0-1,000$ pM OT secreted cytokines (TNF- $\alpha$, IL-6, IL- 8 and IL-1 $\beta$ ) analyzed via Meso Scale. $\mathrm{PI}=30$-min preincubation; $\mathrm{CI}=$ concurrent incubation $(n=1)$.

by seeking to replicate any anti-inflammatory effect of OT in (1) female MOs, and (2) primary MPs from healthy human MP donors. OT has been shown to have systemic anti-inflammatory properties in humans and animals, but the specific mechanism of action is not understood. 
Work in human models has explored MO/MP cells as a potential underpinning mechanism, but the results are inconclusive: Clodi et al. [17] found that in vivo OT attenuates LPS-evoked inflammatory cytokine production in a sample of male participants, but when this team challenged isolated peripheral MOs ex vivo with LPS, no attenuation of cytokine production was observed. Conversely, the work by Szeto et al. [20] suggested that OT does attenuate cytokine production in THP-1-derived MOs and MPs.

We did not observe any consistent anti-inflammatory effect of OT. Although we took care to base our experimental approach on Szeto et al. [20], who reported IL-6 attenuation in THP-1 MPs cotreated with LPS and OT, we were unable to observe a similar pattern in the experiments reported here. The lack of attenuation by OT persisted even after we manipulated numerous factors that could have been masking the inhibitory influences of the hormone, including dosage and timing of administration, the method of cytokine measurement, the use of MOs versus MPs, male versus female participants, and calcium availability for signal transduction. Regardless of the conditions, we did not observe consistent dose-dependent inhibitory influences of OT on cytokine production. These results replicate and extend those obtained by Clodi et al. [17], who did not observe OT effects on cytokine production by LPS-stimulated male MOs cultured ex vivo.

There are a number of reasons for why we and Clodi et al. [17] may have been unable to observe an anti-inflammatory effect of OT. For example, it is always a possibility that cell lines behave differently from normal healthy cells, perhaps being more sensitive to OT than primary human MOs and MPs or due to differences in how OTR is expressed. It is also possible that MPs drive the OT anti-inflammation mechanism and that somehow freezing and thawing compromised MP responses to OT in our experiments. However, this would have to be quite an isolated effect as the MPs showed a high a degree of viability, as evidenced by their marked cytokine production following exposure to LPS. We did not evaluate this possibility here, but future research could explore it as an explanation for the conflicting results.

What does the overall pattern of findings $[6,11,14-17$, $20]$ suggest about the mechanisms underlying the systemic anti-inflammatory properties of OT? Thus far, systemic administration of OT following a variety of immune challenges or injuries has led to reduced inflammation in both animals and humans [e.g., ref. 6-16, 17]. However, incubating LPS-activated primary MOs and MPs from healthy male and female donors with OT ex vivo has yielded no clear, consistent attenuation of inflammatory cytokines. As such, we would argue that the capacity of OT to inhibit cytokine responses to systemically administered LPS [17] are unlikely to be mediated via direct influences on the function of MOs or MPs. Nonetheless, given that its receptor is expressed on these cells [20], OT could regulate some process relevant to $\mathrm{MO} / \mathrm{MP}$ function that was not captured here that may have downstream consequences for systemic inflammation, for example, cell migration. It also remains possible that the effects of OT are dependent on specific micro-environmental conditions not replicated ex vivo. Further research is required to assess which aspects of MO and MP activity are regulated by the OTR and under what conditions. It would be particularly valuable to test whether OT has an effect on the activity of MPs harvested from lymphoid organs or tissues where these cells are resident.

With all that said, it also remains possible that the actions of OT are mainly exerted on other immune cells, like neutrophils, or are secondary to effects on other tissues. For example, vasculature tissue has been shown to express the OTR [20] and respond to systemic and centrally administered OT [27]. OT could regulate the leakiness' of the vasculature at sites of infection or injury, thereby affecting the number of immune cells that can migrate into the tissue or the kinds of activating signals expressed. Adipose tissue is also known to express the OTR [28]. OT dosing in mice has been shown to reduce adipose tissue IL-6 secretion [8], which could also explain decreased systemic inflammation following OT exposure. Another scenario is that OT acts indirectly by regulating the secretion of other hormones with downstream anti-inflammatory effects. For example, some researchers have suggested that OT may cross the blood brain barrier, act on the hypothalamic-pituitary-adrenal axis to increase adenocorticotropic hormone release, which subsequently heightens corticosteroid secretion [29-31]. As such, through regulation of glucocorticoid secretion in the periphery, OT may have consequent anti-inflammatory effects on immune cells. Although this scenario is plausible in rats, in humans, OT seems to have the opposite effect, reducing the systemic output of cortisol [17,29, 31]. As such, in humans at least, the anti-inflammatory effects of OT are probably not solely dependent on the hypothalamic-pituitary-adrenal axis, though other centrally regulated neurohormonal mechanisms could be involved.

There are several limitations to our experiments. First, as is the case with all ex vivo studies, the conditions are 
imperfectly representative of the physiologic environment in which MOs and MPs operate. Second, we focused exclusively on LPS as a stimulus, because Clodi et al. [17] showed that in vivo OT can abrogate its systemic inflammatory effects. However, in the body, MOs and MPs are exposed to a much broader range of threats, not only from pathogens but also from injuries. Third, we used frozen MP matured ex vivo from MOs. Although the MPs were clearly viable, it is possible that the freezing and thawing process compromised their OT responsivity. It is also likely that MPs matured in tissue behave differently from MPs matured ex vivo. It will be important in future research to assess whether OT acts differently on MPs harvested from lymphoid organs or tissues where they are resident. Finally, our experiments involved cells from a fairly small number of individuals $(n=9)$, which precluded the use of formal statistical analyses. However, instead, we calculated and inspected percent differences and found these to be small and/or inconsistent. This, coupled with the lack of a clear attenuation curve in our figures, suggests that here, OT did not inhibit cytokine production consistently, regardless of the donor sex, the dose or the culture conditions. We could have done additional experiments with more subjects, but on the basis of consistently null findings, this seemed unnecessary.

In summary, we conducted a series of experiments to assess whether LPS-activated MOs or MPs showed reduced cytokine production when incubated with OT in vitro and extended previous research by including (1) female MOs, (2) primary human MPs, and (3) by examining cytokine secretion (ELISA and ECL) versus cytokine production (ICS and FACS). Regardless of the conditions, no attenuation in cytokine production by OT was observed in either MOs or MPs. Although OT has been shown to have a systemic anti-inflammatory effect in both animals and humans, based on our work and work by Clodi et al. [17], it appears that this effect is not mediated solely by MP or MO activity. Further research is necessary to understand how OT exerts its anti-inflammatory effect.

\section{Acknowledgements}

This research was supported by grant HD058052 from the National Institute of Child Health and Human Development.

\section{References}

1 Gimpl G, Fahrenholz F: The oxytocin receptor system: structure, function, and regulation. Physiol Rev 2001;81:629-683.

$\checkmark 2$ Carter C, Grippo A, Pournajafinazarloo H, Ruscio M, Porges S: Oxytocin, vasopressin and sociality; in Neumann ID, Landgraf R (eds): Progress in Brain Research. Amsterdam, Elsevier, 2008, vol 170, pp 331-336.

3 Bartz JA, Zaki J, Bolger N, Ochsner KN: Social effects of oxytocin in humans: context and person matter. Trends Cogn Sci 2011;15:301309.

4 Heinrichs M, von Dawans B, Domes G: Oxytocin, vasopressin, and human social behavior. Front Neuroendocrinol 2009;30:548557.

$5 \mathrm{Kemp}$ AH, Guastella AJ: The role of oxytocin in human affect: a novel hypothesis. Curr Dir Psychol Sci 2011;20:222-231.

-6 Iseri SO, Sener G, Saglam B, Gedik N, Ercan F, Yegen BC: Oxytocin protects against sepsis-induced multiple organ damage: role of neutrophils. J Surg Res 2005;126:73-81.

-7 Petersson M, Wiberg U, Lundeberg T, UvnasMoberg K: Oxytocin decreases carrageenan induced inflammation in rats. Peptides 2001; 22:1479-1484.

8 Nation DA, Szeto A, Mendez AJ, Brooks LG, Zaias J, Herderick EE, Gonzales J, Noller CM, Schneiderman N, McCabe PM: Oxytocin at- tenuates atherosclerosis and adipose tissue inflammation in socially isolated ApoE-/mice. Psychosom Med 2010;72:376-382.

$\checkmark$ Ahmed MA, Elosaily GM: Role of oxytocin in deceleration of early atherosclerotic inflammatory processes in adult male rats. Int J Clin Exp Med 2011;4:169-178.

10 Petersson M, Lundeberg T, Sohlstrom A, Wiberg U, Uvnas-Moberg K: Oxytocin increases the survival of musculocutaneous flaps. Naunyn Schmiedebergs Arch Pharmacol 1998; 357:701-704

11 Dusunceli F, Iseri SO, Ercan F, Gedik N, Yegen $\mathrm{C}$, Yegen BC: Oxytocin alleviates hepatic ischemia-reperfusion injury in rats. Peptides 2008;29:1216-1222.

12 Biyikli NK, Tugtepe H, Sener G, VeliogluOgunc A, Cetinel S, Midillioglu S, Gedik N, Yegen BC: Oxytocin alleviates oxidative renal injury in pyelonephritic rats via a neutrophildependent mechanism. Peptides 2006;27: 2249-2257.

13 Tugtepe H, Sener G, Biyikli NK, Yuksel M, Cetinel S, Gedik N, Yegen BC: The protective effect of oxytocin on renal ischemia/reperfusion injury in rats. Regul Pept 2007;140:101108.

14 Iseri SO, Sener G, Saglam B, Gedik N, Ercan F, Yegen BC: Oxytocin ameliorates oxidative colonic inflammation by a neutrophil-de- pendent mechanism. Peptides 2005;26:483491.

15 Iseri SO, Gedik IE, Erzik C, Uslu B, Arbak S, Gedik N, Yegen BC: Oxytocin ameliorates skin damage and oxidant gastric injury in rats with thermal trauma. Burns 2008;34:361369.

16 Iseri SO, Dusunceli F, Erzik C, Uslu B, Arbak $S$, Yegen BC: Oxytocin or social housing alleviates local burn injury in rats. J Surg Res 2010;162:122-131.

17 Clodi M, Vila G, Geyeregger R, Riedl M, Stulnig TM, Struck J, Luger TA, Luger A: Oxytocin alleviates the neuroendocrine and cytokine response to bacterial endotoxin in healthy men. Am J Physiol Endocrinol Metab 2008;295:E686-E691.

18 Miller AH, Maletic V, Raison CL: Inflammation and its discontents: the role of cytokines in the pathophysiology of major depression. Biol Psychiatry 2009;65:732-741.

19 Nathan C, Ding A: Nonresolving inflammation. Cell 2010;140:871-882.

20 Szeto A, Nation DA, Mendez AJ, DominguezBendala J, Brooks LG, Schneiderman N, McCabe PM: Oxytocin attenuates NADPH-dependent superoxide activity and IL-6 secretion in macrophages and vascular cells. Am J Physiol Endocrinol Metab 2008;295:E1495E1501. 
21 Tsuchiya S, Yamabe M, Yamaguchi Y, Kobayashi Y, Konno T, Tada K: Establishment and characterization of a human acute monocytic leukemia cell line (THP-1). Int J Cancer 1980;26:171-176.

22 Carter CS: Oxytocin and sexual behavior. Neurosci Biobehav Rev 1992;16:131-144.

23 Amico JA, Seif SM, Robinson AG: Oxytocin in human plasma: correlation with neurophysin and stimulation with estrogen. J Clin Endocrinol Metab 1981;52:988-993.

24 Kramer KM, Cushing BS, Carter CS, Wu J, Ottinger MA: Sex and species differences in plasma oxytocin using an enzyme immunoassay. Can J Zool 2004;82:1194-1200.
25 Irwin MR, Wang M, Campomayor CO, Collado-Hidalgo A, Cole S: Sleep deprivation and activation of morning levels of cellular and genomic markers of inflammation. Arch Intern Med 2006;166:1756-1762.

26 Cassoni P, Sapino A, Marrocco T, Chini B, Bussolati G: Oxytocin and oxytocin receptors in cancer cells and proliferation. J Neuroendocrinol 2004;16:362-364.

27 Suzuki Y, Satoh S, Kimura M, Oyama H, Asano T, Shibuya M, Sugita K: Effects of vasopressin and oxytocin on canine cerebral circulation in vivo. J Neurosurg 1992;77:424-431.
28 Zingg HH: Vasopressin and oxytocin receptors. Baillieres Clin Endocrinol Metab 1996; 10:75-96.

29 Gibbs DM: Vasopressin and oxytocin: hypothalamic modulators of the stress response: a review. Psychoneuroendocrinology 1986;11: 131-139.

30 Neumann ID: No stress response of the hypothalamo-pituitary-adrenal axis in parturient rats: lack of involvement of brain oxytocin. Endocrinology 2003;144:2473-2479.

31 Jezova D, Skultetyova I, Tokarev DI, Bakos P, Vigas M: Vasopressin and oxytocin in stress. Ann NY Acad Sci 1995;771:192-203. 